\title{
Vortical Structure in Forced Unsteady Circular Jet: Simulation by 3D Vortex Method
}

\author{
M. Kiya, Y. Ido and H. Akiyama \\ Department of Mechanical Engineering \\ Hokkaido University, Sapporo, 060, Japan
}

\begin{abstract}
Three-dimensional vortex-method simulations are made of a round jet forced by axisymmetric, helical and multiple disturbances. The multiple disturbances are combinations of two helical modes rotating in the opposite direction. The present simulations demonstrate the evolution of large-scale vortex structures in the forced jet. Results of the simulations are in reasonable agreement with available experimental results.
\end{abstract}

\section{Introduction}

The vortex structure in the developing region of jets plays a crucial role in the production of Reynolds stresses and the jet noise, and in the mixing of the jet fluid with the surrounding fluid. This indicates that the active control of the vortex structure may have wide applications in fluids engineering. Reynolds and Parekh [23] demonstrated that the round jet experiences a dramatically high rate of spreading by appropriate combinations of frequencies of axisymmetric and orbital disturbances. Experiments conducted with the jet forced by two helical modes rotating in the opposite direction (Kusek et al. [16]) reveal a staggered vortex pattern. The theory and experiments by Cohen and Wygnanski [5] and Long and Petersen [18] showed that the round jet acquires an elliptic or square cross section by a suitable combination of two helical disturbances rotating in the opposite direction. Cotke and Kusek [6] showed that the helical modes pair in the manner discussed by Cohen and Wygnanski [5]. The temporal evolution of a cylindrical jet-like mixing layer excited by axisymmetric and helical disturbances with an additional azimuthal disturbance is numerically studied by Martin and Meiburg [19, 20].

The purpose of this paper is to present results of a three-dimensional vortex-method simulation on the vortex structure in the developing region of a round jet forced by axisymmetric and helical disturbances. Combinations of two helical modes are also studied. No three-dimensional vortex-method simulations of the spatially-developing jet have been published within the authors knowledge, although several simulations are made by finite-difference methods (Grinstein [9], Grinstein et al. [10], Chen et al. [2]). The spatially-evolving jet is expected to include automatically the effect of feedback from the downstream vortex structures on the rolling-up of the jet's shear layer: The disturbance produced by the downstream structures propagates upstream as a pressure wave to be accepted at the edge of the nozzle, affecting the rolling-up process (Ho and Huerre [13], Corke et al. [7]). This feedback effect is not included in the temporally-evolving jet because the periodic boundary condition is employed in the streamwise direction and no sharp edge is included.

The present simulation is made by the use of a vortex-blob core structure proposed by Wincklemans and Leonard [25]. The random vortex method (Chorin [3]) and the particle-exchange scheme (Degond and Mas-Gallic [8]) to represent the viscous diffusion require a huge number of vortex 
blobs for high Reynolds-number flows, which is not always possible for engineering applications. Thus a new method of mimicking the viscous diffusion (Kiya et al. [15]) is employed in this paper. The introduction of this viscous diffusion makes the present vortex method as a turbulence model in the sense that only the dynamics of the large-scale vortices is expected to be approximately simulated. Indeed, as will be shown later, the present simulation has reproduced some of experimental results of the unforced round jet and the jet forced by the helical and multiple disturbances (Long and Petersen [18]).

\section{Numerical method}

An inviscid fluid of uniform density is considered for the moment. The vortex-blob method calculates the evolution of a vorticity field represented by a collection of vortex elements with overlapping cores. The vortex element is referred to as a vortex blob. The vortex-blob method yields a solution which converges to an exact smooth solution of the Euler equations as the density of the blobs is increased and their core size goes to zero subjected to the constraint that the cores overlap (Winckelmans and Leonard [25]).

A vortex blob $\alpha$ is characterized by the position of its center $\mathbf{x}^{\alpha}$, the vorticity $\boldsymbol{\omega}^{\alpha}\left(\mathbf{x}^{\alpha}\right)$, the volume $d \mathbf{x}^{\alpha}$, and the cut-off radius $\sigma_{\alpha}$; the strength $\boldsymbol{\gamma}^{\alpha}$ is defined by $\boldsymbol{\omega}^{\alpha}\left(\mathbf{x}^{\alpha}\right) d \mathbf{x}^{\alpha}$. The vorticity field around the vortex blob $\alpha$ is represented by $\boldsymbol{\omega}^{\alpha}(\mathbf{x})=\sigma_{\alpha}^{-3} p\left(\left|\mathbf{x}-\mathbf{x}^{\alpha}\right|\right)$ where $p(\xi)=(15 / 8 \pi)\left(\xi^{2}+1\right)^{-7 / 2}$ (Winckelmans and Leonard [25]) is a smoothing function. It may be noted that the vortex blob is an element which is introduced to calculate the evolution of a region of distributed vorticity. The vorticity at its center is not the vorticity of flow at that point because the vorticity at an arbitrary position $\mathbf{x}$ is the sum of all the contributions from neighboring vortex blobs. The velocity induced at the center $\mathbf{x}^{\alpha}$ is given by the Biot-Savart law, while its strength $\gamma^{\alpha}$ is given by the Helmholtz vorticity equation. In the present study, the position $\mathbf{x}^{\alpha}$ and the strength $\gamma^{\alpha}$ are updated by the second-order Adams-Bashforth scheme.

Vorticity of vortex blobs generally increases with time. Thus, in order to maintain the spatial resolution, a stretched vortex blob is subdivided into smaller vortex blobs from time to time. One way of doing this is to subdivide a vortex blob of length $l$ and strength $\gamma$ into two blobs of strength $\gamma / 2$ and length $l / 2$ if the length $l$ becomes greater than twice its initial value; the center of the new vortex blobs is $\mathbf{x} \pm(l / 4) \boldsymbol{\gamma} /|\boldsymbol{\gamma}|$. The cut-off radius of the new vortex blobs is chosen as the same as the original one in order to maintain the core overlapping as much as possible. It may be noted, however, that this core-overlapping condition is not always satisfied in regions of flow where the rapid vortex stretching occurs. The subdivision can conveniently be made if a vortex blob a is visualized as a circular cylinder of radius $\delta_{\alpha}$ (which will be referred to as the core radius) and length $l_{\alpha}$. The volume $\Delta \mathbf{x}^{\alpha}=l_{\alpha} \pi \delta_{\alpha}^{2}$ and the circulation $\Gamma_{\alpha}=\left|\boldsymbol{\omega}^{\alpha}\right| \pi \delta_{\alpha}^{2}=\left|\gamma^{\alpha}\right| / l_{\alpha}$ are conserved. This condition and the vorticity equation determine the length $l_{\alpha}$ and the core radius $\delta_{\alpha}$ at each time step of calculation.

In inviscid flow, vorticity tends to diverge within a short time after the start of flow. This implies that the flow cannot be calculated for a sufficiently long time without introducing viscosity or removing vortex blobs which have experienced sufficiently high vortex stretching (Chorin [4]). In viscous flow, however, the vorticity cannot go to infinity because the balance is realized between the intensification of vorticity due to the vortex stretching and the lateral spreading of vorticity by the viscous diffusion. As an example, one considers an initially uniform vorticity distribution which is deformed by an axisymmetric extensional flow. There is a steady state for this flow in which a maximum vorticity $\omega_{\max }$ appears at the axis. If the initial vorticity is uniform with magnitude $\omega_{0}$, within a distance $r_{0}$ from the axis of the extensional flow and zero elsewhere, the condition of 
constant flux across planes normal to the axis gives

$$
\omega_{\max }=\omega_{0}\left(c r_{0}^{2} / 4 \nu\right)
$$

where $c$ is the rate of strain, and $\nu$ implies the kinematic viscosity (Batchelor [1]). As an example, a direct numerical simulation of a round jet at Reynolds number 8000 (Chen et al. [2]) demonstrates that the maximum vorticity, which appears near the center of the shear layer, is less than 3.5 times the initial value.

The above facts have led to an idea that an artificial viscosity may be introduced by limiting the maximum vorticity in the flow within a certain multiple of the initial vorticity $\omega_{0}$ (Kiya et al. [15]). This method of the 'vorticity limiter' is employed in the present study. In terms of vortex blob $\alpha, \omega_{0}$ should be interpreted as $\left|\boldsymbol{\omega}^{\alpha}(t)\right|, r_{0}$ as $\sigma_{\alpha}(t)$, and $c$ as the local rate of strain at $\mathbf{x}^{\alpha}(t)$. Then $\left|\boldsymbol{\omega}^{\alpha}(t+\Delta t)\right|, \Delta t$ being the time step of advancing the flow, should not greater than the value given by equation (1). This is based on the assumption that the steady state is realized within $\Delta t$. However, in the present paper, a simplified version was employed to reduce the computer run time. In the simplified version, $\omega_{0}$ is taken as $\left|\boldsymbol{\omega}^{\alpha}(t=0)\right|$ and the ratio $\left|\boldsymbol{\omega}^{\alpha}(t)\right| /\left|\boldsymbol{\omega}^{\alpha}(t=0)\right|$ is given a constant value for all the vortex blobs. It may be noted in passing that, except for this artificial viscosity, the present method is essentially inviscid in nature. The relation between this artificial viscosity and the molecular viscosity has to be established, in future, by numerical experiments for an analytically accessible problem. However, as will be shown later, large-scale structures in the jets seem to be fairly well represented by this simplified method.

\section{Numerical calculation}

Calculations were performed for a round jet issuing with a velocity $U_{1}$ from a straight circular nozzle of radius $R$ and length $L=0.262 R$ into a surrounding fluid with a velocity $U_{2}$, as shown in Fig. 1. The $x$ axis is the streamwise coordinate, and the $y$ and $z$ axes are the transverse coordinates; the origin is at the center of the exit plane of the nozzle. The vorticity components in the $x, y$ and $z$ directions are denoted by $\omega_{x}, \omega_{y}$ and $\omega_{z}$. The calculations are made for the impulsively starting flow. The external velocity is chosen as $U_{2}=U_{1} / 5$ to have a velocity ratio $\lambda=\left(U_{1}-U_{2}\right) / U_{m}=4 / 3$, where $U_{m}=\left(U_{1}+U_{2}\right) / 2$ is the average velocity. This compound jet is chosen because the evolution of the vortex structure is slower than that of the round jet issuing into the fluid at rest. This property is convenient to study the evolution of vortex structures. The surface of the nozzle is constructed by 96 vortex-blob panels; the circulation of the vortex blobs which construct the panels is determined so as to satisfy the zero cross-flow velocity at reference points on the surface. The jet flow is produced by a source disk in a plane normal to the axis of the nozzle (Fig. 1), which consists of 3791 sources arranged in 30 circular shells of the same thickness. The same nozzle is placed on the opposite side so that the plane which includes the source disk is the plane of symmetry. Outside the source disk, the plane of symmetry is represented by a uniform distribution of source of strength $2 U_{2}$ per unit area to generate the external flow. Only the flow on one side of the plane of symmetry is considered, the flow on the opposite side being the mirror image.

Nascent vortex blobs are introduced into the flow from a point near the edge of the nozzle to simulate the shedding of vorticity. The configuration of a nascent vortex blob and the corresponding vortex panel at the edge of the nozzle are shown in Fig. 2; the edge is defined as the reference point where the normal velocity is set equal to zero. The distance between the edge and the center of the nascent vortex blob is taken as $e=0.061 R$. In order to satisfy Kelvins law of conservation of 
circulation, the circulation of the nascent vortex $\Gamma_{n j}(t)$ at time $\mathrm{t}$ is determined by

$$
\Gamma_{n j}(t)=\Gamma_{p j}(t)-\Gamma_{p j}\left(t-\Delta t_{n}\right)
$$

with $\Gamma_{n j}(0)=\Gamma_{p j}(0) ; \Gamma_{p j}$ is the circulation of the vortex blobs which belong to the $j-$ th panel at the edge of the nozzle, $\Delta t_{n}$ is the time step of introducing the nascent vortices (Hayashi et al. [11]). The nascent vortices are moved by the velocity induced at their center by the panel vortex blobs, the source disk, and the vortex blobs which have been shed from the edge.

The cut-off radius of the vortex blobs of the panels and that of the nascent vortex blobs are chosen as $\sigma / R=0.196$. The time step of advancing the shed vortex blobs is $\Delta t=0.06 R / U_{m}$, while the time step of introducing the nascent vortices is $\Delta t_{n}=0.12 R / U_{m}$. In order to keep the computation time within a reasonable bound, the vortex blobs are removed from the flow field if they have moved more than $20.0 R$ downstream of the edge of the nozzle. This removal was found to have an insignificant effect on the large-scale vortices in the developing region of the jet.

Reynolds number of the jet in the present simulation is unable to be specified although one expects a relation between the value of the limiter and Reynolds number. The vorticity limiter $\omega_{\max } / \omega_{0}=4$ perhaps corresponds to a Reynolds number of several thousands on the basis of the direct numerical simulation by Chen et al. [2].

The calculations were performed for the unexcited jet, and the jet excited by an axisymmetric disturbance, a helical disturbance, and a combination of two helical disturbances rotating in the opposite direction. The disturbances are produced by the sinusoidal oscillation of strength of sources in the outermost two shells of the source disk. The longitudinal velocity fluctuation associated with the disturbance is proportional to $\cos (m \phi-2 \pi f t)$, where $m$ is the mode number, $\phi$ the azimuthal angle measured from the $z$-axis, and $f$ implies the forcing frequency. The mode number $m=0$ implies an axisymmetric disturbance, while non-zero value of $m$ corresponds to helical disturbances. Two modes of helical disturbances $m=1$ and 2 are considered. The multiple disturbances are the combination of $m=+1$ and -1 and of $m=+2$ and -2 , which will be referred to as $m= \pm 1$ and $m= \pm 2$.

The shear layer of the circular jet near the nozzle exit can approximately be regarded as the plane mixing layer. Thus the forcing frequency is chosen as that of the most unstable mode of the plane mixing layer $f \theta / U_{m}=0.035$ (Ho and Huerre [13]), where $\theta$ is the momentum thickness of the shear layer at the exit of the nozzle. In the present calculation the momentum thickness is $R / \theta=$ 18.2 , so that one has $f R /\left(U_{2}-U_{1}\right)=0.48$. The maximum amplitude of the longitudinal velocity fluctuation due to the symmetrical and helical disturbances, which is measured at a radial distance $0.889 R$ from the center of the exit plane of the nozzle, is $0.025 U_{1}$. This implies that the multiple disturbances have the maximum amplitude of $0.050 U_{1}$. The longitudinal velocity fluctuation (which will be referred to as the disturbance velocity) is a stationary wave on the circular shear layer of the jet, being proportional to $\cos (m \phi) \cos (2 \pi f t)$, so that the deformation is fixed at particular azimuthal positions.

\section{Results and discussion}

\subsection{Effect of vorticity limiter on vortex pattern}

Figure 3 compares distributions of vorticity in the unforced round jet for three values of the vorticity limiter $\omega_{\max } / \omega_{0}=4.0,8.0$, and $\infty$ which implies the case where no vorticity limiter is employed. The large-scale vortex structures are almost the same for all values of the limiter except for those in the rightmost large-scale vortices in which finer and finer structures appear as the value of 
the limiter increases. Thus the value $\omega_{\max } / \omega_{0}=4.0$ is employed in the following calculation. As mentioned before, this vorticity limiter may imply that the present simulation corresponds to Reynolds number of the order of several thousands.

It should be noted that, in regions where the core-overlapping of vortex blobs is satisfied, the present result is conjectured to be a reasonable approximation to the solution of the Navier-Stokes equations. The core-overlapping is not realized in the rib regions where the vortex stretching is too large to be covered by the subdivision of the vortex blobs.

\subsection{Unforced jet}

Figure 4 shows the unforced jet visualized by distributions of vortex blobs and the contours of the vorticity components $\omega_{z}$ and $\omega_{y}$, while Fig. 5 is contours of modulus of the vorticity component in the $y z$-plane $\left(\omega_{y}^{2}+\omega_{z}^{2}\right)^{1 / 2}$ and the longitudinal vorticity component $\omega_{x}$. Note that Figs. 4 and 5 are patterns at the same instant of time and thus demonstrate the instantaneous three-dimensional vortex structure in the jet. The longitudinal component of vorticity is produced by the azimuthal deformation of initially circular vortex rings shed from the edge of the nozzle.

The simulated rolling-up of the shear layer of the jet is neither purely axisymmetric nor helical, as shown in Figs. 4 and 5. Within a short time after the start of the flow, however, the rolling-up of the shear layer was found to be axisymmetric; this is reflected in fairly symmetric distributions of vorticity contours in the region $4<x / 2 R<8$, as seen in Figs. 4 and 5 . As time advances, the rolling-up becomes non-axisymmetric. The vorticity distributions of Figs. 4 and 5 might be interpreted as the coexistence of the two modes.

Linear stability analyses of axisymmetric jets with thin initial shear layers $(\theta / 2 R \ll 1)$ such as Mattingly and Chang [21] have predicted that the initial region of the jet is equally unstable to both the axisymmetric $(m=0)$ and the first helical $(m= \pm 1)$ modes. According to Corke et al. [7], the axisymmetric and helical modes of instability rarely, if ever, exist together at the positions $x / 2 R=0.25-0.35$; they switch from one mode type to the other presumably as a result of stochastic disturbances at the lip of the nozzle. However, the present simulation suggests the absence of such mode switching. This point might be interpreted in the following way: The wavelength $(\lambda)$ of the axisymmetric and helical modes in Corke et al. [7] experiment is estimated to be approximately $\lambda / 2 R=0.14$ and 0.12 , respectively, at $x / 2 R=0.25-0.35$. Thus the frequent switching of the modes at these early positions (Corke et al. [7]) can produce vorticity distributions which seem to be a combination of the two modes, if the vorticity distribution is observed over a sufficiently wide region in the longitudinal direction.

In the present simulation, the rolling-up of the shear layer of the unforced jet is initiated by the disturbance which perhaps comes from a very small change in strength of the nascent vortex blobs in the azimuthal direction or from numerical round-off errors. It is also probable that the rolling-up is influenced by the feedback from the downstream vortex structures.

The sudden formation of small-scale vortex structures (downstream of the end of the potential core $x / 2 R \approx 4-5$ ) seems to occur when the first or second merging of the rolled-up vortices takes place, mimicking the transition from the laminar to turbulent flow (Liepmann and Gharib [17]). This is not inconsistent with the experimental observation that the generation of the smallscale turbulence emerges at the first merging of the rolled-up vortices and is completed after the second merging in a two-dimensional excited mixing layer (Huang and Ho [14]). This calculated transition might be affected to some extent by a numerical instability caused by an insufficient core-overlapping between neighboring vortex blobs. 


\subsection{Jet forced by axisymmetric disturbance}

Figure 6 shows the structure of the jet forced by the axisymmetric disturbance. The rolling-up of the shear layer occurs much later than that in the unforced flow. This is also the case in experiments (van Dyke [24]). Furthermore the generation of the longitudinal component of vorticity (not shown here) is much later than the case of the unforced jet; the longitudinal vorticity becomes significant only at positions downstream of $x /(2 R) \approx 4.0-5.0$ where the first vortex merging takes place.

\subsection{Jet forced by helical disturbances}

Figures 7 and 8 show the structure of the jet forced by the helical mode $m=1$. The vorticity contours in the $x y$ and $x z$ planes (Fig. 7) are generally antisymmetric in the region $x / 2 R<3.5$ in such a manner that the dominant vortex structure is helical. The helical structure is formed by successive helical pairing of elemental vortex rings shed from the exit of the nozzle (Fig. 8). The flow visualization of the forced jet by smoke particles clearly shows the existence of helical vortices (Reeds [22]).

The growth rate of the jet in the early region $x / 2 R<2$ is basically similar in the unforced jet and the jet forced by the axisymmetric and helical $(m=1)$ modes. This is consistent with the prediction of the linear stability analyses that the axisymmetric $(m=0)$ and helical $(m=1)$ modes have almost the same growth rate. However, as a result of nonlinear effects, the streamwise lifespan of the helical mode is less than that of the axisymmetric mode.

The breakdown to turbulence for the helical mode $m=1$ occurs approximately at the same streamwise position as the unforced case. On the other hand, the forcing by the double-helical mode $m=2$ (not shown here) shifted the position of the breakdown to turbulence more upstream than that by the mode $m=1$. This is because the the azimuthal deformation of the vortex rings due to the forcing has a shorter wavelength for $m=2$ than for $m=1$. The shorter wavelength corresponds to a higher local curvature, leading to a higher rate of the vortex stretching. This probably led to the earlier breakdown to turbulence.

\subsection{Jet forced by multiple disturbances}

Results for $m= \pm 1$ are presented in Fig. 9. In this multiple mode the amplitude of the disturbance velocity takes a maximum on the $z$ axis at $z= \pm R(\phi=0$ and $\pi)$, while nodes of the disturbance velocity exist on the $y$ axis at $y= \pm R(\phi=\pi / 2$ and $3 \pi / 2)$. Thus the vortex merging due to the forcing is almost confined to the azimuthal positions of the maximum amplitude of the disturbance velocity. As a result, the spreading of the jet becomes much greater in the $x z$ plane than in the $x y$ plane in a region $x / 2 R \geq 4$, yielding an ellipse-like cross section of the jet. However, the difference in the growth rate of the jet in both planes is insignificant in the early region $x / 2 R \leq 3$. This is consistent with the experiment of Long and Petersen [18].

One might conjecture that the ellipse-like cross section is produced by the same mechanism as the axis switching of the jet issuing from a 2:1 elliptic nozzle (Ho and Gutmark [12]) due to the self-induction distortion of a vortex filament associated with non-uniform curvature. It seems unlikely that self-induction distortion plays an important role in this forced jet. In the present case the jet is initially circular and the shear layer is modulated in thickness in the circumferential direction. The different shear-layer thickness in the azimuthal direction corresponds to different local frequencies and growth rates of the shear layer, producing the ellipse-like cross section (Long and Petersen [18]).

The jet forced by the multiple disturbance $m= \pm 2$ is presented in Figs. 10 and 11. In this case the amplitude of the disturbance velocity attains a maximum at four circumferential positions: 
$\phi=0$ and $\pi(z= \pm R$ on the $z$ axis $)$, and $\phi=\pi / 2$ and $3 \pi / 2(y= \pm R$ on the $y$ axis $)$. This disturbance produces a square-like cross section of the jet at some distances downstream of the nozzle exit, as shown in Fig. 11. The growth of the jet in the diagonal sections (Fig. 10a) is greatest among all the forced cases. The square-like cross section is experimentally demonstrated by Long and Petersen [18]. Moreover, the vorticity distribution in the diagonal yz plane is in reasonable agreement with the phase-averaged vorticity distribution of Long and Petersen [18] in which small-scale vorticity components are smeared out by the phase-averaging, see Fig. 12.

Finally, the relation between the spatially-evolving and temporally-evolving calculations will be discussed. As mentioned before, a temporally-evolving calculation such as Martin and Meiburg [19, 20] does not include the effect of feedback from the downstream vortex structures. We are in the opinion that the rapid development of the three-dimensional structures in the early part of the shear layer of the unforced jet (Figs. 4 and 5) is partly associated with the feedback effect, because a direct numerical simulation of a temporally-evolving jet has shown that the three-dimensional structure hardly develops in the axisymmetric mode without artificial three-dimensional disturbances (Y. Miyake 1996, private communication). Moreover, the periodic boundary condition in the longitudinal direction for the temporally-evolving calculation is reasonable only if several rolling-up vortices of similar structure are arranged in the spatially-evolving jet. This is approximately the case for the unforced jet (Fig. 4) and the jets forced with $m=0$ (Fig. 6) and $m=1$ (Fig. 7). However, for the combined forcing $m= \pm 2$ (Fig. 10), the rapid change in the vortex structure in the longitudinal direction throws doubt on the accuracy of the time-evolving calculation.

\section{Concluding remarks}

The present paper has described results of the three-dimensional vortex-blob simulations on the round jet forced by axisymmetric, helical and multiple disturbances. The rolling-up of the initial shear layer of the unforced jet is neither purely axisymmetric nor helical, suggesting a combination of the axisymmetric and helical modes of instability. The axisymmetric disturbance produces highly coherent vortex rings, leading to the minimal growth rate of the jet. On the other hand, the combination of two double-helical disturbances $m= \pm 2$ yields the greatest spreading rate among all the cases studied in this paper. These numerical results are in fairly good agreement with available experimental results. Thus the 'vorticity limiter' algorithm employed in the present study is expected to be useful in calculating large-scale vortex structures in free turbulent shear flows although its accuracy should be examined by numerical experiments for analytically accessible problems in future.

Issues to be tackled in future are to obtain combinations of axisymmetric and helical disturbances which yields a maximal growth rate, probably including azimuthal corrugations of the nozzle.

\section{References}

[1] Batchelor, G.K., An Introduction to Fluid Dynamics, Cambridge University Press, UK, 1967.

[2] Chen, J.H., Lienau, J.J. and Kollmann, W., "Numerical Simulation of Low Re-Number Turbulence in Round Jets," Turbulent Shear Flows, 9, pp. 123-145, 1995.

[3] Chorin, A.J., "Numerical Study of Slightly Viscous Flow," J. Fluid Mech., 57, pp. 785-796, 1973. 
[4] Chorin, A.J., "Hairpin Removal in Vortex Interactions," J. Comp. Phys., 91, pp. 1-21, 1990.

[5] Cohen J. and Wygnanski, I., "The Evolution of Instabilities in the Axisymmetric Jet, Part 1. The Linear Growth of Disturbances Near the Nozzle," J. Fluid Mech., 176, pp. 191-235, 1987.

[6] Corke, T.C. and Kusek, S.M., "Resonance in Axisymmetric Jets with Controlled Helical-Mode Input," J. Fluid Mech., 249, pp. 307-336, 1993.

[7] Corke, T.C., Shakib, F. and Nagib, H.M., "Effects of Low Amplitude Forcing on Axisymmetric Jet Flows," J. Fluid Mech., 223, pp. 253-311, 1991.

[8] Degond, P. and Mas-Gallic, S., "The Weighted Particle Method for Convection-Diffusion Equations. Part 1: The Case of an Isotropic Viscosity," Math. Comp., 53, pp. 485-507, 1989.

[9] Grinstein, F.F., "Vorticity Dynamics in Spatially-Developing Rectangular Jets," AIAA Paper 93-3286, 1993.

[10] Grinstein, F.F., Gutmark, E. and Parr, T., "Near Field Dynamics of Subsonic Free Square Jets. A Computational and Experimental Study," Phys. Fluids, 7, pp. 1483-1497, 1995.

[11] Hayashi, R., Kida, T. and Yasutomi, Z., "Numerical Simulation of Outer Flow Around an ACV by Discrete Vortex Methods," J. Japan. Soc. Aeron. Astron. Sci., 39, pp. 258-267 (in Japanese), 1991.

[12] Ho, C.-M. and Gutmark, J., "Vortex Induction and Mass Entrainment in a Small Aspect-Ratio Jet," Fluid Mech., 179, pp. 383-405, 1987.

[13] Ho, C.-M. and Huerre, P., "Perturbed free shear layers," Ann. Rev. Fluid Mech., 16, pp. 365424, 1984.

[14] Huang, L.-S. and Ho, C.-M., "Small-Scale Transition in a Plane Mixing Layer," J. Fluid Mech., 210, pp. 475-500, 1990.

[15] Kiya, M., Toyoda, K., Ishii, H., Kitamura, M. and Ohe, T., "Numerical Simulation and FlowVisualization Experiment on Deformation of Pseudo-Elliptic Vortex Rings," Fluid Dyn. Res., 10, pp. 117-131, 1992.

[16] Kusek, C.M., Corke, T.C. and Reithenthel, P., "Control of Two and Three-Dimensional Modes in the Initial Region of an Axisymmetric Jet," AIAA Paper 89-0968, 1989.

[17] Liepmann, D. and Gharib, M., "The Role of Streamwise Vorticity in the Near-Field Entrainment of Round Jet," J. Fluid Mech., 245, pp. 643-668, 1992.

[18] Long, T.A. and Petersen, R.A., "Controlled Interactions in a Forced Axisymmetric Jet, Part 1. The Distortion of the Mean Flow," J. Fluid Mech., 235, pp. 37-55, 1992.

[19] Martin, J.E. and Meiburg, E., "Numerical Investigation of Three-Dimensionally Evolving Jets Subject to Axisymmetric and Azimuthal Perturbation," J. Fluid Mech., 230, pp. 271-318, 1991.

[20] Martin, J.E. and Meiburg, E., "Numerical Investigation of Three-Dimensionally Evolving Jets Under Helical Perturbations," J. Fluid Mech., 243, pp. 457-487, 1992.

[21] Mattingly, G.E. and Chang, C.C., "Unstable Waves on an Axisymmetric Jet Column," J. Fluid Mech., 65, pp. 541-560, 1974. 
[22] Reeds, H., "Gallery of Fluid Motion," Phys. Fluids A, 1, pp. 1439-1450, 1989.

[23] Reynolds, W.C. and Parekh, D.E., "Control of Structure in Turbulent Flows," AFOSR-TR-880036, Thermosciences Division, Department of Mechanical Engineering, Stanford University, 1987.

[24] van Dyke, M., An Album of Fluid Motion, The Parabolic Press, Stanford, CA, 1982.

[25] Winckelmans, G.S. and Leonard, A., "Contributions to Vortex Particle Methods for the Computation of Three-Dimensional Incompressible Unsteady Flows," J. Comp. Phys., 109, pp. 247-273, 1993.

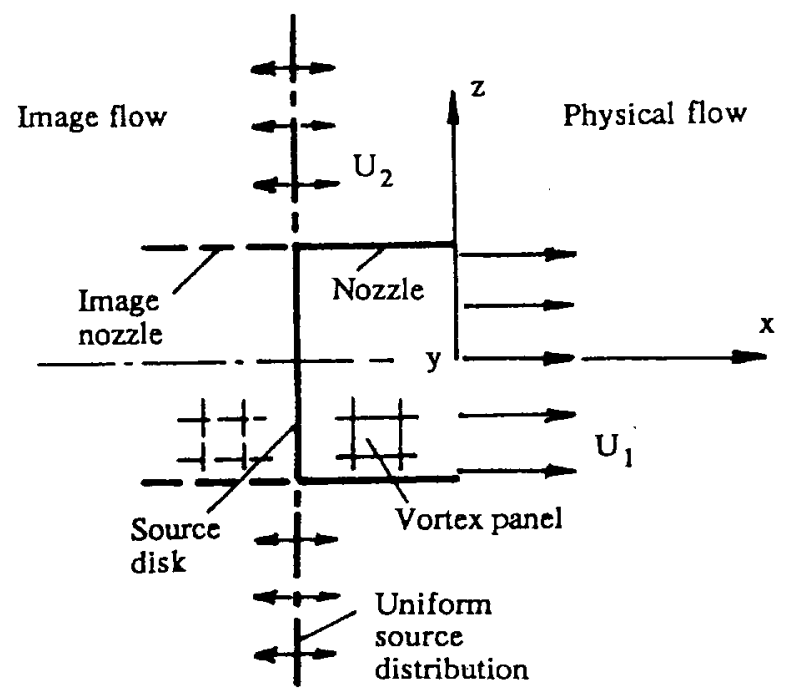

Figure 1: Configuration of nozzle, coordinate system and definition of symbols. 


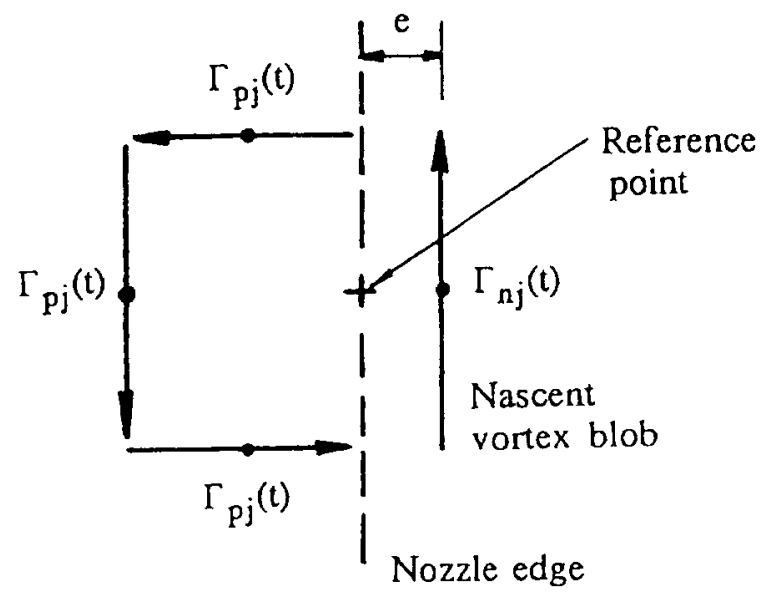

Figure 2: Vortex panel at exit of nozzle and nascent vortex blob.
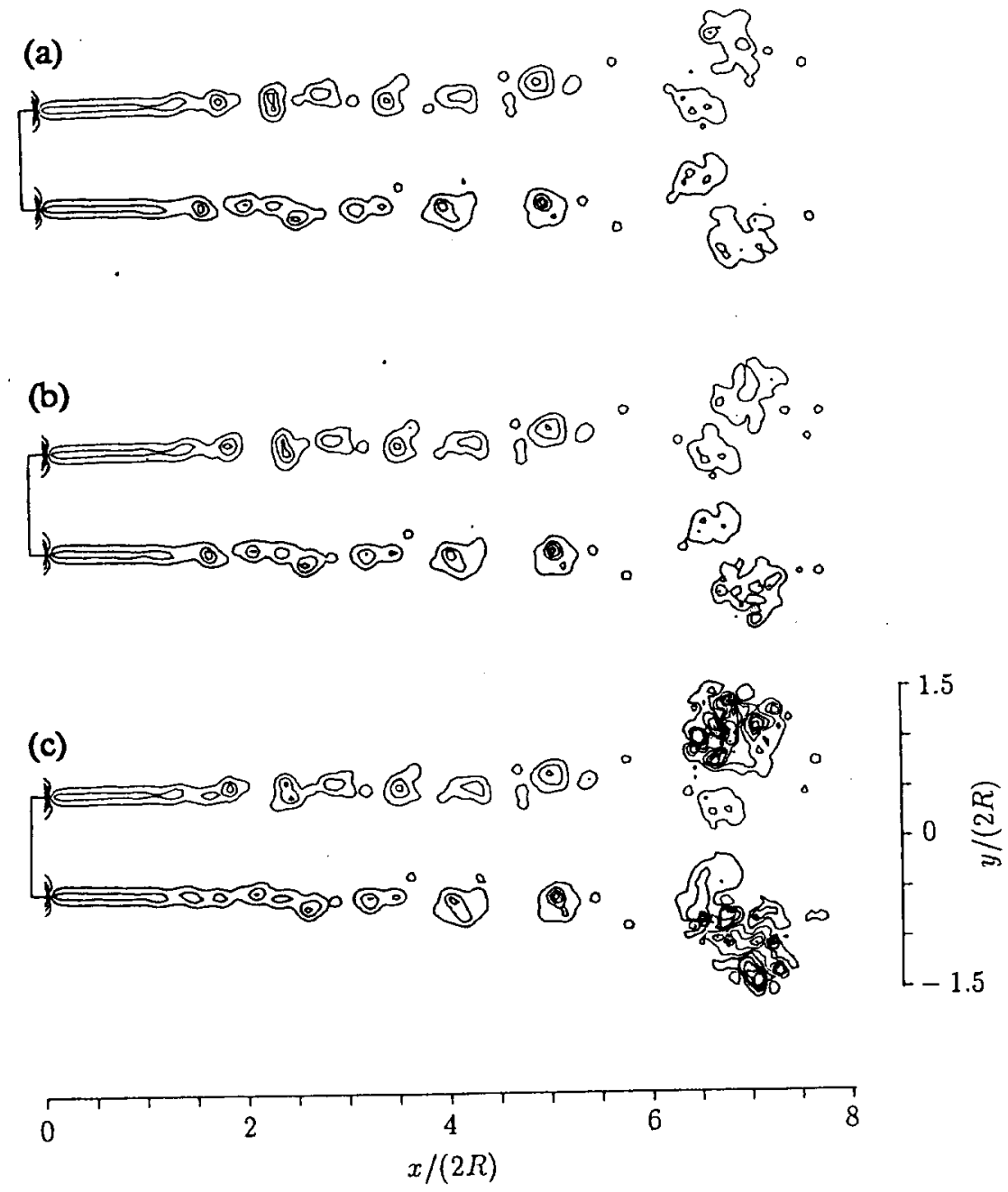

Figure 3: Contours of vorticity component $\omega_{z}$ of round jet for different values of vorticity limiter at time $U_{m} t / R=16.2$. (a) $\omega_{\max } / \omega_{0}=4.0$, (b) 8.0 , and (c) $\infty$. 

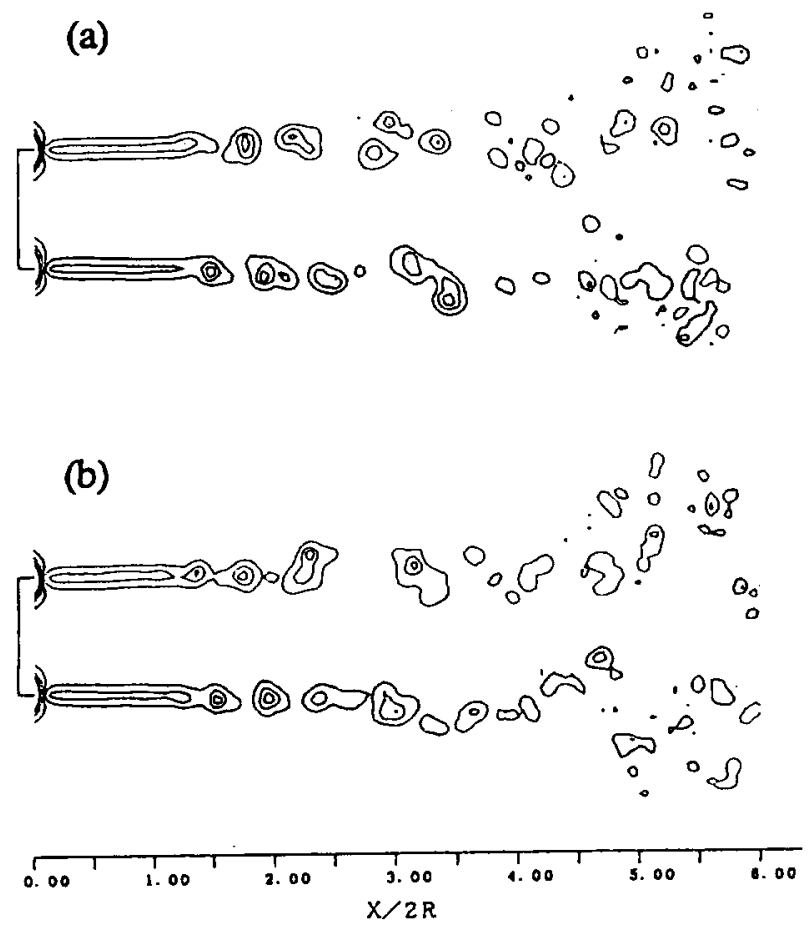

Figure 4: Contours of vorticity component (a) $\omega_{z}$ in $x y$ plane and (b) $\omega_{y}$ in $x z$ plane of unforced jet at $U_{m} t / R=30.0$. 


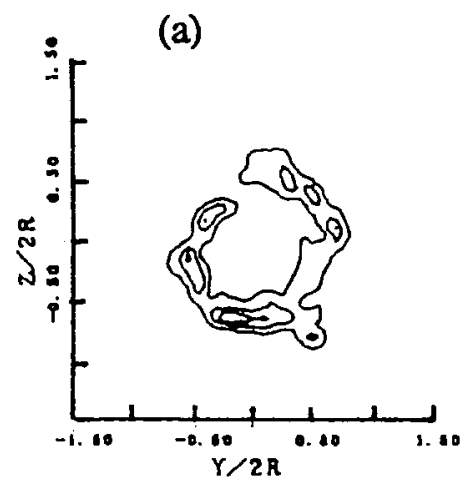

(b)

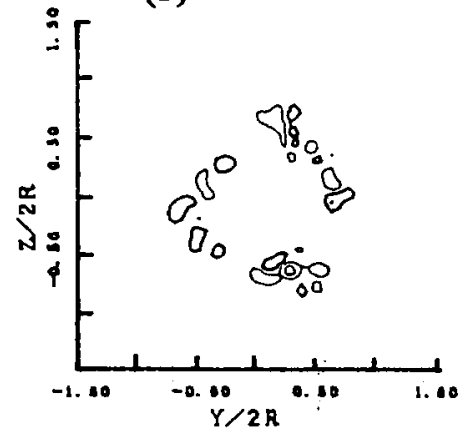

Figure 5: Contours of vorticity component (a) $\left(\omega_{y}^{2}+\omega_{z}^{2}\right)^{1 / 2}$ and (b) $\omega_{x}$ of unforced jet in cross section at $x / 2 R=3.0$ at $U_{m} t / R=30.0$.
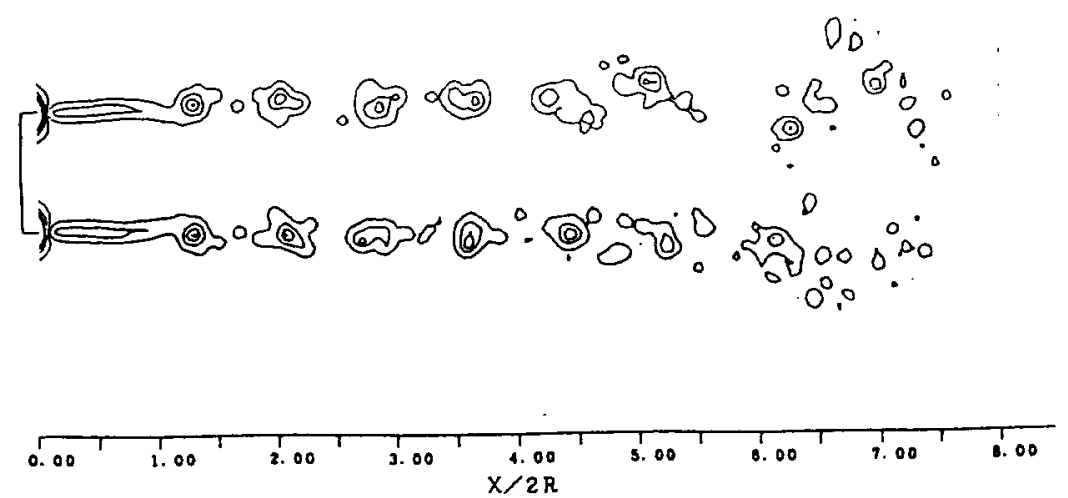

Figure 6: Contours of vorticity component $\omega_{y}$ in $x z$ plane of jet forced by axisymmetric disturbance $m=0 . U_{m} t / R=30.0$. 

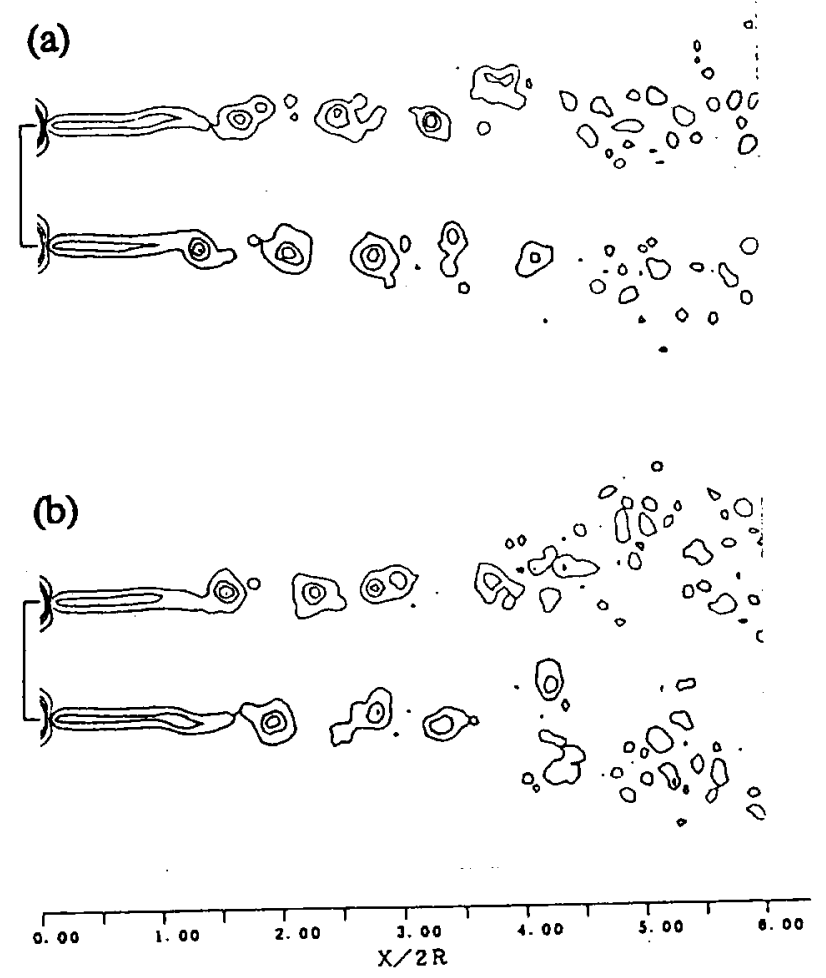

Figure 7: Structure of jet forced by helical disturbance $m=1$ visualized by contours of vorticity component (a) $\omega_{z}$ in $x y$ plane and (b) $\omega_{y}$ in $x z$ plane at $U_{m} t / R=30.0$. 

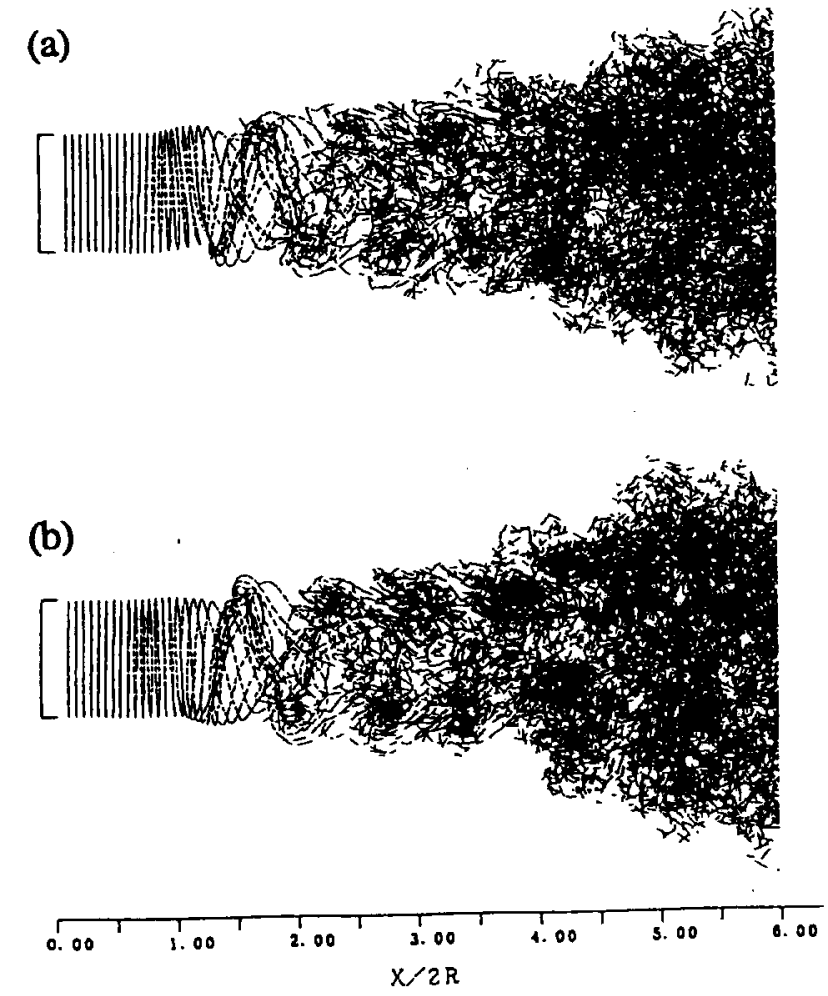

Figure 8: Structure of jet forced by helical disturbance $m=1$ visualized by vortex blobs at $U_{m} t / R=30.0$. All vortex blobs viewed from (a) $z$ direction and (b) $y$ direction are shown. 

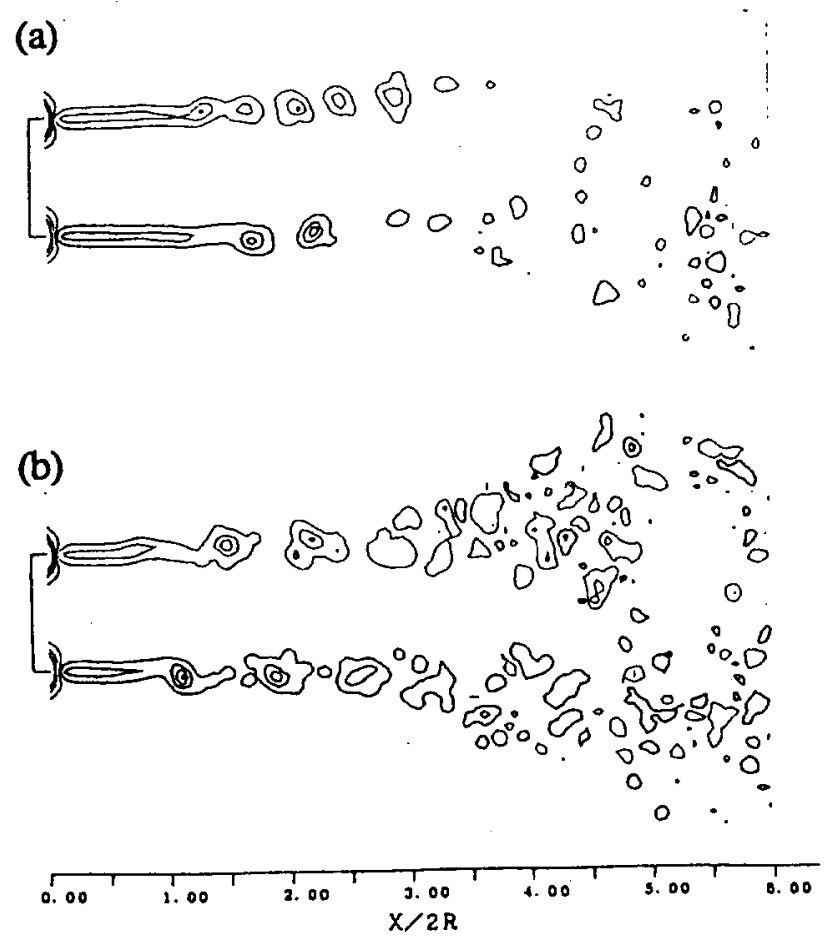

Figure 9: Structure of jet forced by multiple disturbance $m= \pm 1$ visualized by contours of vorticity component (a) $\omega_{z}$ in $x y$ plane and (b) $\omega_{y}$ in $x z$ plane at $U_{m} t / R=28.8$. 

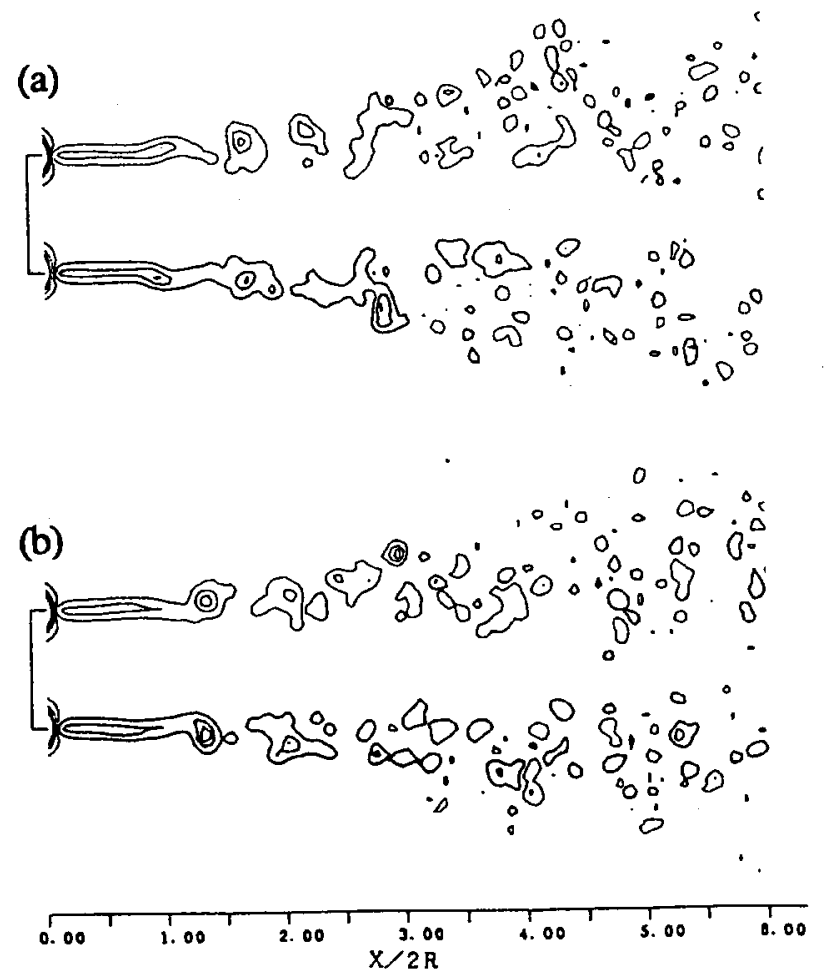

Figure 10: Structure of jet forced by multiple disturbance $m= \pm 2$ visualized by contours of vorticity component (a) $\omega_{z}$ in $x y$ plane and (b) $\omega_{y}$ in $x z$ plane at $U_{m} t / R=30.0$. 

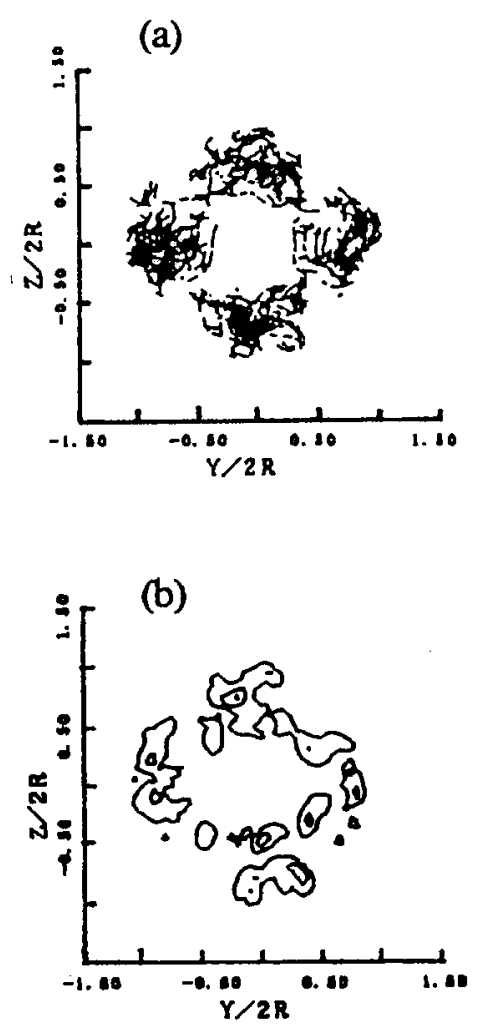

Figure 11: Cross-sectional structure of jet forced by $m= \pm 2$ visualized by (a) vortex blobs in region $2.5<x / 2 R<3.0$ and (b) contours of vorticity component $\left(\omega_{y}^{2}+\omega_{z}^{2}\right)^{1 / 2}$ at $x / 2 R=3.0$. $U_{m} t / R=30.0$. 
(a)
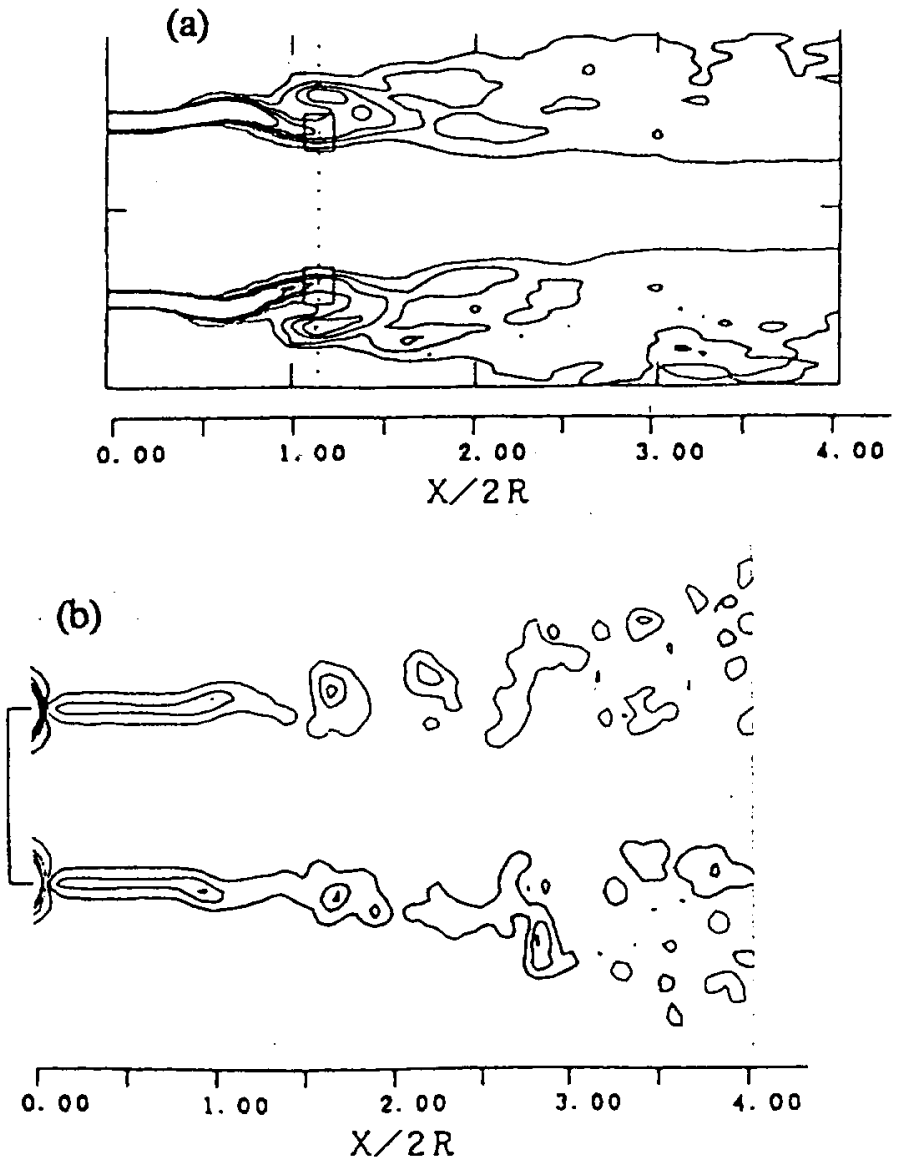

Figure 12: Contours of vorticity component $\omega_{z}$ in $x y$ plane of jet forced by $m= \pm 2$. (a) Experiment by Long and Petersen [18], (b) present simulation. Origin of (b) is shifted to left for purpose of comparison because phase of forcing is different for (a) and (b). 